\title{
Rate of Approximation for Modified Lupaş-Jain-Beta Operators
}

\author{
M. Qasim, ${ }^{1}$ Asif Khan $\mathbb{C D}^{2}{ }^{2}$ Zaheer Abbas, ${ }^{1}$ Princess Raina, ${ }^{1}$ and Qing-Bo Cai ${ }^{3}{ }^{3}$ \\ ${ }^{1}$ Department of Mathematical Sciences, Baba Ghulam Shah Badshah University, Rajouri, 185234 Jammu and Kashmir, India \\ ${ }^{2}$ Department of Mathematics, Aligarh Muslim University, Aligarh-202002, India \\ ${ }^{3}$ School of Mathematics and Computer Science, Quanzhou Normal University, Quanzhou 362000, China
}

Correspondence should be addressed to Qing-Bo Cai; qbcai@126.com

Received 12 May 2020; Revised 17 June 2020; Accepted 13 July 2020; Published 3 August 2020

Guest Editor: Bipan Hazarika

Copyright (c) 2020 M. Qasim et al. This is an open access article distributed under the Creative Commons Attribution License, which permits unrestricted use, distribution, and reproduction in any medium, provided the original work is properly cited.

The main intent of this paper is to innovate a new construction of modified Lupaş-Jain operators with weights of some Beta basis functions whose construction depends on $\sigma$ such that $\sigma(0)=0$ and $\inf _{x \in[0, \infty)} \sigma^{\prime}(x) \geq 1$. Primarily, for the sequence of operators, the convergence is discussed for functions belong to weighted spaces. Further, to prove pointwise convergence Voronovskaya type theorem is taken into consideration. Finally, quantitative estimates for the local approximation are discussed.

\section{Introduction}

In 1972, Jain [1] with the help of Poisson distribution introduced a famous linear positive operators as follows:

$$
P_{m}^{[\alpha]}(f ; x)=\sum_{j=0}^{\infty} \mathscr{W}_{\alpha}(j, m x) f\left(\frac{j}{m}\right), \quad x \geq 0,
$$

where $m \geq 1, f$ defined on $[0, \infty)$, and

$$
\begin{gathered}
\mathscr{W}_{\alpha}(j, m x)=m x(m x+j \alpha)^{j-1} \frac{e^{-(m x+j \alpha)}}{j !}, \quad 0 \leq \alpha<1, \\
\sum_{j=0}^{\infty} \mathscr{W}_{\alpha}(j, m x)=1 .
\end{gathered}
$$

If we put $\alpha=0$ in (1), then it becomes Szász-Mirakyantype.

In 1995, Lupaş [2] introduced a sequence of linear positive operators; later on in 1999, it was modified by Agratini [3] as follows:

$$
L_{m}(f ; x)=2^{-m x} \sum_{j=0}^{\infty} \frac{(m x)_{j}}{j ! 2^{j}} f\left(\frac{j}{m}\right), \quad x \geq 0
$$

and also discussed the Kantorovich and Durrmeyer variant of operator (1).

In 2018, Tunca et al. [4] modified operator (3) in such a way that in the construction, authors take the negative subscript -1 of the Pochhammer symbol into consideration; due to this, the calculations become simpler in a remarkable degree just as

$$
L_{m}^{\alpha}(f ; x)=2^{-(m x+j \alpha)} \sum_{j=0}^{\infty} \frac{m x(m x+1+j \alpha)}{j-1} f\left(\frac{j}{m}\right), \quad x \geq 0
$$

In order to approximate Lebesgue integrable functions, the most important modifications are Kantorovich and Durrmeyer integral operators. The Durrmeyer variant of operator (1) is introduced by Tarabie [5] and Mishra and Patel [6] with some beta basis functions.

In 2011, Cárdenas-Morales et al. [7] defined the Bernstein-type operators by $B_{m}\left(f o \sigma^{-1}\right) o \sigma$ and also presents a better degree of approximation depending on $\sigma$. This type of approximation operators generalizes the Korovkin set from $\left\{e_{0}, e_{1}, e_{2}\right\}$ to $\left\{e_{0}, \sigma, \sigma^{2}\right\}$. The Durrmeyer variant of $B_{m}\left(f o \sigma^{-1}\right) o \sigma$ is defined in [8]. In 2014, Aral et al. [9] defined a similar modification of Szász-Mirakyan-type operators by using a suitable function $\sigma$. 
Motivated by the above mentioned work very recently, Bodur [10] introduced a new modification of operator (4) by using a suitable function $\sigma$, which satisfies the following properties:

$\left(\sigma_{1}\right) \sigma$ be a continuously differentiable function on $[0, \infty)$

$\left(\sigma_{2}\right) \sigma(0)=0$ and $\inf _{x \in[0, \infty)} \sigma^{\prime}(x) \geq 1$

The new formulated operators are defined as

$$
\begin{aligned}
\mathscr{L}_{m, \sigma}^{\alpha}(f ; x)= & 2^{-(m \sigma(x)+j \alpha)} \sum_{j=0}^{\infty} \frac{m \sigma(x)(m \sigma(x)+1+j \alpha)_{j-1}}{2^{j} j !} \\
& \cdot\left(f o \sigma^{-1}\right)\left(\frac{j}{m}\right), \quad x \geq 0,
\end{aligned}
$$

for $m \geq 1, x \geq 0$, and suitable functions $f$ defined on $[0, \infty)$.

As we know, in order to approximate Lebesgue integrable functions, the most important modifications are Kantorovich and Durrmeyer integral operators. Motivated by the above mentioned Durrmeyer type generalizations of various operators and also from [11-23], in this paper, Durrmeyer-type modification of generalized Lupaş-Jain operators (5) by taking weights of some beta basis function is defined as follows:

$$
\begin{aligned}
\mathscr{D}_{m, \sigma}^{\alpha}(f ; x)= & 2^{-(m \sigma(x)+j \alpha)} \sum_{j=0}^{\infty} \frac{m \sigma(x)(m \sigma(x)+1+j \alpha)_{j-1}}{2^{j} j !} \\
& \int_{0}^{\infty} b_{m, j}(\vartheta)\left(f o \sigma^{-1}\right)(\vartheta) d \vartheta
\end{aligned}
$$

where $m \in \mathbb{N}$ and $b_{m, j}(\vartheta)$ is defined as

$$
b_{m, j}(\vartheta)=\frac{1}{\beta(m+1, j)} \frac{\vartheta^{j-1}}{(1+\vartheta)^{m+j+1}},
$$

where $\beta(m+1, j)$ is the beta basis function and $\sigma$ is a function satisfying the conditions $\left(\sigma_{1}\right)$ and $\left(\sigma_{2}\right)$ given above.

The rest of the work is organized as follows: in the second section, moments and central moments for $\mathscr{D}_{m, \sigma}^{\alpha}$ are calculated. In the third section, we study convergence properties of $\mathscr{D}_{m, \sigma}^{\alpha}$ in the light of weighted space. In the fourth section, we obtain the order of approximation of new constructed operators associated with the weighted modulus of continuity. In the fifth section, we shall prove Voronovskaya-type theorem in quantitative form. These kinds of results are very useful to describe the rate of point-wise convergence. Finally, in the last section, we obtain some local approximation results related to $\mathscr{K}$-functional.

\section{Basic Results}

In this section, we prove some lemmas for $\mathscr{D}_{m, \sigma}^{\alpha}$ which are required to prove our main results.

Lemma 1. Let $\mathscr{D}_{m, \sigma}^{\alpha}$ be given by (6). Then for each $x \geq 0, m$ $\in \mathbb{N}$, and $0 \leq \alpha<1$, we have

(i) $\mathscr{D}_{m, \sigma}^{\alpha}(1 ; x)=1$ (ii) $\mathscr{D}_{m, \sigma}^{\alpha}(\sigma ; x)=\sigma(x) / 1-\alpha$

(iii) $\mathscr{D}_{m, \sigma}^{\alpha}\left(\sigma^{2} ; x\right)=\left(1 /(m-1)(1-\alpha)^{2}\right)\left(m \sigma^{2}(x)+\left(\alpha^{2}-2\right.\right.$ $\alpha+3 /(1-\alpha)) \sigma(x))$

(iv) $\mathscr{D}_{m, \sigma}^{\alpha}\left(\sigma^{3} ; x\right)=\left(1 /(m-1)(m-2)(1-\alpha)^{3}\right)\left(m^{2} \sigma^{3}(x)+\right.$ $\left(\left(6 m+3 m(1-\alpha)^{2}\right) / 1-\alpha\right) \sigma^{2}(x)+(6(\alpha+1)+6(1-$ $\left.\left.\alpha)^{2}+2(1-\alpha)^{4} /(1-\alpha)^{2}\right) \sigma(x)\right)$

(v) $\mathscr{D}_{m, \sigma}^{\alpha}\left(\sigma^{4} ; x\right)=\left(1 /(m-1)(m-2)(m-3)(1-\alpha)^{4}\right)\left(m^{3}\right.$ $\sigma^{4}(x)+\left(\left(12 m^{2}+6 m^{2}(1-\alpha)^{2}\right) / 1-\alpha\right) \sigma^{3}(x)+(24 m$ $\left.\alpha+36 m+36 m(1-\alpha)^{2}+11 m(1-\alpha)^{4} /(1-\alpha)^{2}\right) \sigma^{2}(x)$ $+\left(26 \alpha^{2}+68 \alpha+24+(36 \alpha+36)(1-\alpha)^{2}+22(1-\right.$ $\left.\left.\alpha)^{4}+6(1-\alpha)^{6} /(1-\alpha)^{3}\right) \sigma(x)\right)$

By using beta function and Lemma 2.1 in [4], it can be proved. So we omit it. Now, from the linearity of the operators $\mathscr{D}_{m, \sigma}^{\alpha}$, we can state Lemma 2.

Lemma 2. For operators $\mathscr{D}_{m, \sigma}^{\alpha}$, we have the following properties:

(i) $\mathscr{D}_{m, \sigma}^{\alpha}(\sigma(\vartheta)-\sigma(x) ; x)=(\alpha / 1-\alpha) \sigma(x)$

(ii) $\mathscr{D}_{m, \sigma}^{\alpha}\left((\sigma(\vartheta)-\sigma(x))^{2} ; x\right)=\left(m /(m-1)(1-\alpha)^{2}-1+\right.$ $\alpha / 1-\alpha) \sigma^{2}(x)+\left(\alpha^{2}-2 \alpha+3 /(m-1)(1-\alpha)^{3}\right) \sigma(x)$

(iii) $\mathscr{D}_{m, \sigma}^{\alpha}\left((\sigma(\vartheta)-\sigma(x))^{4} ; x\right)=(1 /(m-1)(m-2)(m-3)$ $\left.(1-\alpha)^{4}\right)\left(\left\{m^{3}+6 m(m-2)(m-3)(1-\alpha)^{2}-4 m^{2}\right.\right.$ $(m-3)(1-\alpha)-4(m-1)(m-2)(m-3)(1-\alpha)^{3}+$ $\left.(m-1)(m-2)(m-3)(1-\alpha)^{4}\right\} \sigma^{4}(x)+\left\{6 \alpha^{2}-12 \alpha\right.$ $+18(m-2)(m-3)(1-\alpha)-\left(24 m+12 m(1-\alpha)^{2}\right)$ $\left.(m-3)+\left(12 m^{2}+6 m^{2}(1-\alpha)^{2} /(1-\alpha)\right)\right\} \sigma^{3}(x)+$ $\left\{24 m \alpha+36 m+36 m(1-\alpha)^{2}+11 m(1-\alpha)^{4} /(1-\alpha)^{2}\right.$ $-\left(24(\alpha+1)+24(1-\alpha)^{2}+8(1-\alpha)^{4}\right)(m-3) / 1-$ $\alpha\} \sigma^{2}(x)+\left(26 \alpha^{2}+68 \alpha+24+(36 \alpha+36)(1-\alpha)^{2}+\right.$ $\left.\left.22(1-\alpha)^{4}+6(1-\alpha)^{6} /(1-\alpha)^{3}\right) \sigma(x)\right)$

\section{Convergence of $\mathscr{D}_{m, \sigma}^{\alpha}$}

Here, we prove the convergence of $\mathscr{D}_{m, \sigma}^{\alpha}$ by using weight function. Let $\lambda(x)$ be a function satisfying the conditions $\left(\sigma_{1}\right)$ and $\left(\sigma_{2}\right)$ given above. Also, let $\lambda(x)=1+\sigma^{2}(x)$ be a weight function and the weighted space is defined as follows:

$$
\mathscr{B}_{\lambda}[0, \infty)=\left\{f:[0, \infty) \longrightarrow \mathbb{R}|| f(x) \mid \leq \mathscr{M}_{f} \lambda(x), x \geq 0\right\}
$$

where $\mathscr{M}_{f}$ is a constant which depends only on $f$, with the norm

$$
\|f\|_{\lambda}=\sup _{x \in[0, \infty)} \frac{|f(x)|}{\lambda(x)} .
$$


Also, we mention some subspaces of $\mathscr{B}_{\lambda}[0, \infty)$ as

$\mathscr{C}_{\lambda}[0, \infty)=\left\{f \in \mathscr{B}_{\lambda}[0, \infty): f\right.$ is continuous on $\left.[0, \infty)\right\}$,

$\mathscr{C}_{\lambda}^{*}[0, \infty)=\left\{f \in \mathscr{C}_{\lambda}[0, \infty): \lim _{x \rightarrow \infty} \frac{f(x)}{\lambda(x)}=\mathscr{M}_{f}=\right.$ constant $\}$,

$U_{\lambda}[0, \infty)=\left\{f \in \mathscr{C}_{\lambda}[0, \infty): \frac{f(x)}{\lambda(x)}\right.$ is uniformly continuous on $\left.[0, \infty)\right\}$.

It is obvious that $\mathscr{C}_{\lambda}^{*}[0, \infty) \subset U_{\lambda}[0, \infty) \subset \mathscr{C}_{\lambda}[0, \infty) \subset$ $\mathscr{B}_{\lambda}[0, \infty)$.

We have the following results for the weighted Korovkintype theorems due to Gadjiev [24]

Lemma 3. [24].

The positive linear operators $\mathscr{T}_{m}, m \geq 1$ act from $\mathscr{C}_{\lambda}$ $[0, \infty)$ to $\mathscr{B}_{\lambda}[0, \infty)$ if and only if the inequality

$$
\left|\mathscr{T}_{m}(\lambda ; x)\right| \leq \mathscr{M}_{m} \lambda(x), \quad x \geq 0
$$

holds, where $\mathscr{M}_{m}>0$ is a constant depending on $\mathrm{m}$.

Theorem 4 (see [24]). Let the sequence of positive linear operators $\mathscr{T}_{m}, m \geq 1$ acting from $\mathscr{C}_{\lambda}[0, \infty)$ to $\mathscr{B}_{\lambda}[0, \infty)$ and satisfying

$$
\lim _{m \rightarrow \infty}\left\|\mathscr{T}_{m} \sigma^{r}-\sigma^{r}\right\|_{\lambda}=0, \quad r=0,1,2
$$

Then, for each $f \in C_{\lambda}^{*}[0, \infty)$, we have

$$
\lim _{m \rightarrow \infty}\left\|\mathscr{T}_{m}(f)-f\right\|_{\lambda}=0
$$

Remark 5. Examining Lemma 1 based on the famous Korovkin theorem [25], it is clear that $\left(\mathscr{D}_{m, \sigma}^{\alpha}\right)_{m>1}$ does not form an approximation process. Now, in order to obtain convergence properties, we replace the constant $\alpha$ by $\alpha_{m} \in[0,1)$ such that $\lim _{m \rightarrow \infty} \alpha_{m}=0$.

Theorem 6. Let $0 \leq \alpha_{m}<1$ such that $\lim _{m \rightarrow \infty} \alpha_{m}=0$, and also, let $\mathscr{D}_{m, \sigma}^{\alpha}$ be the sequence of positive linear operators. Then, for each function $f \in C_{\lambda}^{*}[0, \infty)$, we have

$$
\lim _{m \rightarrow \infty}\left\|\mathscr{D}_{m, \sigma}^{\alpha_{m}}(f)-f\right\|_{\lambda}=0
$$

Proof. From Lemma 1, we obtain

$$
\begin{gathered}
\left\|\mathscr{D}_{m, \sigma}^{\alpha_{m}}(1 ; x)-1\right\|_{\lambda}=0, \\
\left\|\mathscr{D}_{m, \sigma}^{\alpha_{m}}(\sigma ; x)-\sigma\right\|_{\lambda} \leq \frac{\alpha_{m}}{1-\alpha_{m}}, \\
\left\|\mathscr{D}_{m, \sigma}^{\alpha}\left(\sigma^{2}\right)-\sigma^{2}\right\|_{\lambda} \leq \frac{m-(m-1)\left(1-\alpha_{m}\right)^{2}}{(m-1)\left(1-\alpha_{m}\right)^{2}}+\frac{\alpha^{2}-2 \alpha_{m}+3}{(m-1)\left(1-\alpha_{m}\right)^{3}} .
\end{gathered}
$$

Hence, by Theorem 4, we deduce

$$
\lim _{m \rightarrow \infty}\left\|\mathscr{D}_{m, \sigma}^{\alpha}(f)-f\right\|_{\lambda}=0
$$

\section{Rate of Convergence}

In this part, we would like to determine the rate of convergence for $\mathscr{D}_{m, \sigma}^{\alpha}$ by weighted modulus of continuity $\omega_{\sigma}(f ; \delta)$ which was introduced by Holhos [26] in 2008, as follows:

$$
\omega_{\sigma}(; \delta)=\sup _{x, \vartheta \in[0, \infty),|\sigma(\vartheta)-\sigma(x)| \leq \delta} \frac{|f(\vartheta)-f(x)|}{\lambda(\vartheta)+\lambda(x)}, \quad \delta>0,
$$

where $f \in \mathscr{C}_{\lambda}[0, \infty)$, with the following properties:

(i) $\omega_{\sigma}(f ; 0)=0$

(ii) $\omega_{\sigma}(f ; \lambda) \geq 0, \lambda \geq 0$, for $f \in \mathscr{C}_{\lambda}[0, \infty)$

(iii) $\lim _{\lambda \rightarrow 0} \omega_{\sigma}(f ; \lambda)=0$, for each $f \in U_{\lambda}[0, \infty)$

Theorem 7 (see [26]). Let $\mathscr{T}_{m}: \mathscr{C}_{\lambda}[0, \infty) \rightarrow \mathscr{B}_{\lambda}[0, \infty)$ be a sequence of positive linear operators with

$$
\begin{aligned}
& \left\|\mathscr{T}_{m}\left(\sigma^{0}\right)-\sigma^{0}\right\|_{\lambda^{0}}=a_{m}, \\
& \left\|\mathscr{T}_{m}(\sigma)-\sigma\right\|_{\lambda^{1 / 2}}=b_{m}, \\
& \left\|\mathscr{T}_{m}\left(\sigma^{2}\right)-\sigma^{2}\right\|_{\lambda}=c_{m}, \\
& \left\|\mathscr{T}_{m}\left(\sigma^{3}\right)-\sigma^{3}\right\|_{\lambda^{3 / 2}}=d_{m},
\end{aligned}
$$

where the sequences $a_{m}, b_{m}, c_{m}$, and $d_{m}$ converge to zero as $m \longrightarrow \infty$. Then

$$
\left\|\mathscr{T}_{m}(f)-f\right\|_{\lambda^{3 / 2}} \leq\left(7+4 a_{m}+2 c_{m}\right) \omega_{\sigma}\left(f ; \lambda_{m}\right)+\|f\|_{\lambda} a_{m},
$$

for all $f \in \mathscr{C}_{\lambda}[0, \infty)$, where

$$
\lambda_{m}=2 \sqrt{\left(a_{m}+2 b_{m}+c_{m}\right)\left(1+a_{m}\right)}+a_{m}+3 b_{m}+3 c_{m}+d_{m} .
$$

Theorem 8. Let $0 \leq \alpha<1$ such that $\lim _{m \rightarrow \infty} \alpha_{m}=0$, and also, let $\mathscr{D}_{m, \sigma}^{\alpha}$ be the sequence of positive linear operators. Then for all $f \in C_{\lambda}[0, \infty)$, we have

$$
\begin{aligned}
\left\|\mathscr{D}_{m, \sigma}^{\alpha_{m}}(f)-f\right\|_{\lambda^{3 / 2}} \leq & \left(7+\frac{2 m-2(m-1)\left(1-\alpha_{m}\right)^{2}}{(m-1)\left(1-\alpha_{m}\right)^{2}}\right. \\
& \left.+\frac{2 \alpha_{m}^{2}-4 \alpha_{m}+6}{(m-1)\left(1-\alpha_{m}\right)^{3}}\right) \omega_{\sigma}\left(f ; \delta_{m}\right),
\end{aligned}
$$


where

$$
\begin{aligned}
\delta_{m}= & 2 \sqrt{\frac{2 \alpha_{m}}{1-\alpha_{m}}+\frac{m-(m-1)\left(1-\alpha_{m}\right)^{2}}{(m-2)\left(1-\alpha_{m}\right)^{2}}+\frac{\alpha_{m}^{2}-2 \alpha_{m}+3}{(m-1)\left(1-\alpha_{m}\right)^{3}}} \\
& +\frac{3 \alpha_{m}}{1-\alpha_{m}}+\frac{3 m-3(m-1)\left(1-\alpha_{m}\right)^{2}}{(m-1)\left(1-\alpha_{m}\right)^{2}}+\frac{3 \alpha_{m}^{2}-6 \alpha_{m}+6}{(m-1)\left(1-\alpha_{m}\right)^{3}} \\
& +\frac{m^{2}-(m-1)(m-2)\left(1-\alpha_{m}\right)^{3}}{(m-1)(m-2)\left(1-\alpha_{m}\right)^{3}}+\frac{6 m^{2}-3 m\left(1-\alpha_{m}\right)^{2}}{(m-1)(m-2)\left(1-\alpha_{m}\right)^{4}} \\
& +\frac{2\left(1-\alpha_{m}\right)^{4}+6\left(1-\alpha_{m}\right)^{2}+6\left(\alpha_{m}+1\right)}{(m-1)(m-2)\left(1-\alpha_{m}\right)^{5}} .
\end{aligned}
$$

Proof. We should calculate the sequences $\left(a_{m}\right),\left(b_{m}\right),\left(c_{m}\right)$, and $\left(d_{m}\right)$, in order to apply Theorem 7 . In light of Lemma 1 , clearly, we have

$$
\begin{aligned}
& a_{m}=\left\|\mathscr{D}_{m, \sigma}^{\alpha_{m}}\left(\sigma^{0}\right)-\sigma^{0}\right\|_{\lambda^{0}}=0, \\
& b_{m}=\left\|\mathscr{D}_{m, \sigma}^{\alpha_{m}}(\sigma)-\sigma\right\|_{\lambda^{1 / 2}} \leq \frac{\alpha_{m}}{1-\alpha_{m}}, \\
& c_{m}=\left\|\mathscr{D}_{m, \sigma}^{\alpha_{m}}\left(\sigma^{2}\right)-\sigma^{2}\right\|_{\lambda} \leq \frac{m-(m-1)\left(1-\alpha_{m}\right)^{2}}{(m-1)\left(1-\alpha_{m}\right)^{2}}+\frac{\alpha_{m}^{2}-2 \alpha_{m}+3}{(m-1)\left(1-\alpha_{m}\right)^{3}} .
\end{aligned}
$$

Finally,

$$
\begin{aligned}
d_{m}= & \left\|\mathscr{D}_{m, \sigma}^{\alpha_{m}}\left(\sigma^{3}\right)-\sigma^{3}\right\|_{\lambda^{3 / 2}} \leq \frac{m^{2}-(m-1)(m-2)\left(1-\alpha_{m}\right)^{3}}{(m-1)(m-2)\left(1-\alpha_{m}\right)^{3}} \\
& +\frac{6 m-3 m\left(1-\alpha_{m}\right)^{2}}{(m-1)(m-2)\left(1-\alpha_{m}\right)^{4}} \\
& +\frac{2\left(1-\alpha_{m}\right)^{4}+6\left(1-\alpha_{m}\right)^{2}+6\left(\alpha_{m}+1\right)}{(m-1)(m-2)\left(1-\alpha_{m}\right)^{5}}
\end{aligned}
$$

Thus, Theorem 7 is satisfied. Hence, we have the desired result.

Remark 9. For $\lim _{\lambda \rightarrow 0} \omega_{\sigma}(f ; \lambda)=0$ in Theorem 8 , we obtain

$$
\lim _{m \rightarrow \infty}\left\|\mathscr{D}_{m, \sigma}^{\alpha_{m}}(f)-f\right\|_{\lambda^{3 / 2}}=0, \quad \text { for } f \in U_{\lambda}[0, \infty)
$$

\section{Pointwise Convergence of $\mathscr{D}_{m, \sigma}^{\alpha}$}

In this section, we shall analyze pointwise convergence of $\mathscr{D}_{m, \sigma}^{\alpha}$ by obtaining the Voronovskaya theorem in a quantitative form by using the same technique in [7].

Theorem 10. Let $0 \leq \alpha<1$ such that $\lim _{m \rightarrow \infty} \alpha_{m}=0$, and also, let $f \in \mathscr{C}_{\lambda}[0, \infty), x \in[0, \infty)$ and suppose that $\left(f \circ \sigma^{-1}\right)^{\prime}$ and $\left(f \circ \sigma^{-1}\right)^{\prime \prime}$ exist at $\sigma(x)$. If $\left(f \circ \sigma^{-1}\right)^{\prime \prime}$ is bounded on $[0, \infty)$, then, we have

$$
\lim _{m \rightarrow \infty} m\left[\mathscr{D}_{m, \sigma}^{\alpha}(f ; x)-f(x)\right]=\frac{3 \sigma(x)\left(f o \sigma^{-1}\right)^{\prime \prime} \sigma(x)}{2}
$$

Proof. By using Taylor expansion of $\left(f \circ \sigma^{-1}\right)$ at $\sigma(x) \in[0, \infty)$, we have

$$
\begin{aligned}
f(\vartheta)= & \left(f \circ \sigma^{-1}\right)(\sigma(\vartheta)) \\
= & \left(f \circ \sigma^{-1}\right)(\sigma(x))+\left(f o \sigma^{-1}\right)^{\prime}(\sigma(x)(\sigma(\vartheta)-\sigma(x))) \\
& +\frac{\left(f \circ \sigma^{-1}\right)^{\prime \prime}(\sigma(x))(\sigma(\vartheta)-\sigma(x))^{2}}{2} \\
& +\lambda_{x}(\vartheta)(\sigma(\vartheta)-\sigma(x))^{2},
\end{aligned}
$$

where

$$
\lambda_{x}(\vartheta)=\frac{\left(f \circ \sigma^{-1}\right)^{\prime \prime}(\sigma(\vartheta))-\left(f o \sigma^{-1}\right)^{\prime \prime}(\sigma(x))}{2} .
$$

Therefore, (28) together with the assumption on $f$ ensures that

$$
\left|\lambda_{x}(\vartheta)\right| \leq \mathscr{K}, \quad \text { for all } \vartheta \in[0, \infty)
$$

and is convergent to zero as $\vartheta \rightarrow x$. Now applying the operators (6) to the equality (27), we obtain

$$
\begin{aligned}
& {\left[\mathscr{D}_{m, \sigma}^{\alpha}(f ; x)-f(x)\right]=\left(f o \sigma^{-1}\right)^{\prime}(\sigma(x)) \mathscr{D}_{m, \sigma}^{\alpha}((\sigma(\vartheta)-\sigma(x)) ; x)} \\
& \quad+\frac{\left(f o \sigma^{-1}\right)^{\prime \prime}(\sigma(x)) \mathscr{D}_{m, \sigma}^{\alpha}\left((\sigma(\vartheta)-\sigma(x))^{2} ; x\right)}{2} \\
& \quad+\mathscr{D}_{m, \sigma}^{\alpha}\left(\lambda^{x}(\vartheta)\left((\sigma(\vartheta)-\sigma(x))^{2} ; x\right)\right) .
\end{aligned}
$$

From Lemma 2, we get

$$
\begin{array}{r}
\lim _{m \rightarrow \infty} m \mathscr{D}_{m, \sigma}^{\alpha}((\sigma(\vartheta)-\sigma(x)) ; x)=0, \\
\lim _{m \rightarrow \infty} m \mathscr{D}_{m, \sigma}^{\alpha}\left((\sigma(\vartheta)-\sigma(x))^{2} ; x\right) \leq 3 \sigma(x) .
\end{array}
$$

By estimating the equality (30), we will get the proof.

Since from (28), for every $\varepsilon>0, \lim _{\vartheta \rightarrow x} \lambda_{x}(\vartheta)=0$. Let $\delta>0$ such that $\left|\lambda_{x}(\vartheta)\right|<\varepsilon$ for every $\vartheta \geq 0$. By Cauchy-Schwartz inequality, we get

$$
\begin{gathered}
\lim _{m \rightarrow \infty} m \mathscr{D}_{m, \sigma}^{\alpha}\left(\left|\lambda_{x}(\vartheta)\right|(\sigma(\vartheta)-\sigma(x))^{2} ; x\right) \\
\leq \varepsilon \lim _{m \rightarrow \infty} m \mathscr{D}_{m, \sigma}^{\alpha}\left((\sigma(\vartheta)-\sigma(x))^{2} ; x\right) \\
+\frac{\mathscr{K}}{\delta^{2}} \lim _{m \rightarrow \infty} \mathscr{D}_{m, \sigma}^{\alpha}\left((\sigma(\vartheta)-\sigma(x))^{4} ; x\right) .
\end{gathered}
$$

Since

$$
\lim _{m \rightarrow \infty} m \mathscr{D}_{m, \sigma}^{\alpha}\left((\sigma(\vartheta)-\sigma(x))^{4} ; x\right)=0,
$$


we obtain

$$
\lim _{m \rightarrow \infty} m \mathscr{D}_{m, \sigma}^{\alpha}\left(\left|\lambda_{x}(\vartheta)\right|(\sigma(\vartheta)-\sigma(x))^{2} ; x\right)=0 .
$$

Thus, by taking into account equations (31), (32), and (35) to equation (30), the proof is completed.

Remark 11. If we choose $\alpha_{n}=1 /\left((m+1)^{a}\right)$ with $a>1$, then, one can easily see that $\lim _{m \rightarrow \infty} \alpha_{m}=0$ and $m \lim _{m \rightarrow \infty} \alpha_{m}=0$.

\section{Local Approximation}

In this section, for the operators $\mathscr{D}_{m, \sigma}^{\alpha}$, we shall present local approximation theorems. Let $\mathscr{C}_{B}[0, \infty)$ denote the space of real-valued continuous and bounded functions $f$ defined on the interval $[0, \infty)$. The norm $\|\cdot\|$ on the space $\mathscr{C}_{B}[0, \infty)$ is defined by

$$
\|f\|=\sup _{0 \leq x<\infty}|f(x)| .
$$

$\mathscr{K}$-functional is defined as

$$
\mathscr{K}_{2}(f, \delta)=\inf _{s \in W^{2}}\left\{\|f-s\|+\delta\left\|f^{\prime}\right\|\right\},
$$

where $\delta>0$ and $W^{2}=\left\{s \in \mathscr{C}_{B}[0, \infty): s^{\prime}, s^{\prime \prime} \in \mathscr{C}_{B}[0, \infty)\right\}$. By Devore and Lorentz ([27], p. 177, Theorem 6.4), there exists an absolute constant $\mathscr{C}>0$ such that

$$
\mathscr{K}(f, \delta) \leq \mathscr{C} \omega_{2}(f, \sqrt{\delta}) .
$$

The second order modulus of smoothness is as follows:

$$
\omega_{2}(f, \sqrt{\delta})=\sup _{0<h \leq \sqrt{\delta} x \in[0, \infty)} \sup _{0, \infty}|f(x+2 h)-2 f(x+h)+f(x)|,
$$

where $f \in C_{B}[0, \infty)$. The usual modulus of continuity of $f \epsilon$ $C_{B}[0, \infty)$ is defined by

$$
\omega(f, \delta)=\sup _{0<h \leq \delta x \in[0, \infty)} \sup _{0, \infty}|f(x+h)-f(x)| .
$$

Theorem 12. Let $0 \leq \alpha<1$ such that $\lim _{m \rightarrow \infty} \alpha_{m}=0$ and for all $f \in \mathscr{C}_{B}[0, \infty)$. Also, let $\sigma$ be a function satisfying the conditions $\left(\sigma_{1}\right)$ and $\left(\sigma_{2}\right)$, and $\left\|\sigma^{\prime \prime}\right\|$ is finite. Then, there exists an absolute constant $\mathscr{C}>0$ such that

$$
\left|\mathscr{D}_{m, \sigma}^{\alpha}(f ; x)-f(x)\right| \leq \mathscr{C} \mathscr{K}\left(f, \delta_{m}(x)\right),
$$

where

$$
\delta_{m}(x)=\left(\frac{m}{(m-1)(1-\alpha)^{2}}-\frac{1+\alpha}{1-\alpha}\right) \sigma^{2}(x)+\frac{\alpha^{2}-2 \alpha+3}{(m-1)(1-\alpha)^{3}} \sigma(x) .
$$

Proof. Let $s \in W^{2}$ and $x, \vartheta \in[0, \infty)$. By Taylor's formula, we have

$$
\begin{aligned}
s(\vartheta)= & s(x)+\left(s o \sigma^{-1}\right)^{\prime}(\sigma(x))(\sigma(\vartheta)-\sigma(x)) \\
& +\int_{\sigma(x)}^{\sigma(\vartheta)}(\sigma(\vartheta)-v)\left(s o \sigma^{-1}\right)^{\prime \prime}(v) d v .
\end{aligned}
$$

By using the equality,

$$
\left(s o \sigma^{-1}\right)^{\prime \prime}(\sigma(x))=\frac{s^{\prime \prime}(x)}{\left(\sigma^{\prime}(x)\right)^{2}}-s^{\prime^{\prime}}(x) \frac{\sigma^{\prime \prime}(x)}{\left(\sigma^{\prime}(x)\right)^{3}} .
$$
get

Now, putting $v=\sigma(x)$ in the last term in equality (43), we

$$
\begin{aligned}
& \int_{\sigma(x)}^{\sigma(\vartheta)}(\sigma(\vartheta)-v)\left(s o \sigma^{-1}\right)^{\prime^{\prime}}(v) d v \\
& =\int_{x}^{\vartheta}(\sigma(\vartheta)-\sigma(x))\left[\frac{s^{\prime \prime}(x) \sigma^{\prime}(x)-s^{\prime}(x) \sigma^{\prime \prime}(v)}{\left(\sigma^{\prime}(x)\right)^{2}}\right] d x \\
& =\int_{\sigma(x)}^{\sigma(\vartheta)}(\sigma(\vartheta)-v) \frac{s^{\prime^{\prime}}\left(\sigma^{-1}(v)\right)}{\left(\sigma^{\prime}\left(\sigma^{-1}(v)\right)\right)^{2}} d v \\
& \quad-\int_{\sigma(x)}^{\sigma(\vartheta)}(\sigma(\vartheta)-v) \frac{s^{\prime}\left(\sigma^{-1}(v)\right) \sigma^{\prime \prime}\left(\sigma^{-1}(v)\right)}{\left(\sigma^{\prime}\left(\sigma^{-1}(v)\right)\right)^{3}} d v
\end{aligned}
$$

By applying operator (6) to the both sides of equality (43) and from Lemma 1 , we deduce

$$
\begin{aligned}
& \mathscr{D}_{m, \sigma}^{\alpha}(s ; x)=s(x) \\
& +\mathscr{D}_{m, \sigma}^{\alpha}\left(\int_{\sigma(x)}^{\sigma(\vartheta)}(\sigma(\vartheta)-v) \frac{s^{\prime \prime}\left(\sigma^{-1}(v)\right)}{\left(\sigma^{\prime}\left(\sigma^{-1}(v)\right)\right)^{2}} d v ; x\right) \\
& -\mathscr{D}_{m, \sigma}^{\alpha}\left(\int_{\sigma(x)}^{\sigma(\vartheta)}(\sigma(\vartheta)-v) \frac{s^{\prime}\left(\sigma^{-1}(v)\right) \sigma^{\prime \prime}\left(\sigma^{-1}(v)\right)}{\left(\sigma^{\prime}\left(\sigma^{-1}(v)\right)\right)^{3}} d v ; x\right) .
\end{aligned}
$$

As we know, $\sigma$ is strictly increasing on $[0, \infty)$, and with condition $\left(\sigma_{2}\right)$, we get

$$
\left|\mathscr{D}_{m, \sigma}^{\alpha}(s ; x)-s(x)\right| \leq \mathscr{M}_{m, 2}^{\sigma}(x)\left(\left\|s^{\prime^{\prime}}\right\|+\left\|s^{\prime}\right\|\left\|\sigma^{\prime^{\prime}}\right\|\right),
$$

where

$$
\mathscr{M}_{m, 2}^{\sigma}(x)=\mathscr{D}_{m, \sigma}^{\alpha}\left((\sigma(\vartheta)-\sigma(x))^{2} ; x\right)
$$


Also,

$$
\left|\mathscr{D}_{m, \sigma}^{\alpha}\right| \leq\|f\|
$$

Hence, we have

$$
\begin{aligned}
& \left|\mathscr{D}_{m, \sigma}^{\alpha}(f ; x)-f(x)\right| \\
& \quad \leq\left|\mathscr{D}_{m, \sigma}^{\alpha}(f-s ; x)\right|+\left|\mathscr{D}_{m, \sigma}^{\alpha}(s ; x)-s(x)\right|+|s(x)-f(x)| \\
& \quad \leq 2\|f-s\|+\left\{\left(\frac{m}{(m-1)(1-\alpha)^{2}}-\frac{1+\alpha}{1-\alpha}\right) \sigma^{2}(x)\right. \\
& \left.\quad+\frac{\alpha^{2}-2 \alpha+3}{(m-1)(1-\alpha)^{3}} \sigma(x)\right\}\left(\left\|s^{\prime^{\prime}}\right\|+\left\|s^{\prime}\right\|\left\|\sigma^{\prime^{\prime}}\right\|\right),
\end{aligned}
$$

if $\mathscr{C}=\max \left\{2,\left\|\sigma^{\prime \prime}\right\|\right\}$; then

$$
\begin{aligned}
& \left|\mathscr{D}_{m, \sigma}^{\alpha}(f ; x)-f(x)\right| \\
& \quad \leq \mathscr{C}\left(2\|f-s\|+\left\{\left(\frac{m}{(m-1)(1-\alpha)^{2}}-\frac{1+\alpha}{1-\alpha}\right) \sigma^{2}(x)\right.\right. \\
& \left.\left.\quad+\frac{\alpha^{2}-2 \alpha+3}{(m-1)(1-\alpha)^{3}} \sigma(x)\right\}\left\|s^{\prime}\right\|_{W^{2}}\right) .
\end{aligned}
$$

Taking infimum over all $s \in W^{2}$, we obtain

$$
\left|\mathscr{D}_{m, \sigma}^{\alpha}(f ; x)-f(x)\right| \leq \mathscr{C} \mathscr{K}\left(f, \delta_{m}(x)\right) .
$$

Theorem 12 is proved.

\section{Data Availability}

The data used to support the findings of this study are included within the article.

\section{Conflicts of Interest}

We declare that there is no conflict of interest.

\section{Acknowledgments}

The first author is grateful to the Council of Scientific and Industrial Research (CSIR), India, for providing the Senior Research Fellowship with file no. 09/1172(0001)/2017EMR-I. This work is also supported by the Project for High-level Talent Innovation and Entrepreneurship of Quanzhou (Grant No. 2018C087R), the Program for New Century Excellent Talents in Fujian Province University, and Sponsoring Agreement for Overseas Studies in Fujian Province.

\section{References}

[1] G. C. Jain, "Approximation of functions by a new class of linear operators," Journal of the Australian Mathematical Society, vol. 13, no. 3, pp. 271-276, 1972.

[2] A. Lupaş, "The approximation by some positive linear operators," in Proceedings of the International Dortmund meeting on Approximation Theory, pp. 201-229, Akademie Verlag: Berlin, Germany, 1995.

[3] O. Agratini, "On a sequence of linear and positive operators," Facta Universitatis, Series: Mathematics and Informatics, vol. 14, pp. 41-48, 1999.

[4] B. Tunca, G. Bodur, and D. Soylemez, "On Lupaş-Jain operators," Studia Universitatis Babes-Bolyai Matematica, vol. 63, no. 4, pp. 525-537, 2018.

[5] S. Tarabie, "On Jain-beta linear operators," Applied Mathematics \& Information Sciences, vol. 6, no. 2, pp. 213-216, 2012.

[6] V. N. Mishra and P. Patel, "Some approximation properties of modified Jain-Beta operators," Journal of Calculus of Variations, vol. 2013, Article ID 489249, 8 pages, 2013.

[7] D. Cárdenas-Morales, P. Garrancho, and I. Rasa, "Bernsteintype operators which preserve polynomials," Computers \& Mathematcs with Applications, vol. 62, no. 1, pp. 158-163, 2011.

[8] T. Acar, A. Aral, and I. Rasa, "Modified Bernstein-Durrmeyer operators," General Mathematics, vol. 22, no. 1, pp. 27-41, 2014.

[9] A. Aral, D. Inoan, and I. Rasa, "On the generalized SzászMirakyan operators," Results in Mathematics, vol. 65, no. 3-4, pp. 441-452, 2014.

[10] M. Bodur, "Modified Lupaş-Jain operators," Mathematica Slovaca, vol. 70, no. 2, pp. 431-440, 2020.

[11] T. Acar, A. M. Acu, and N. Manav, "Approximation of functions by genuine Bernstein-Durrmeyer type operators," Journal of Mathematical Inequalities, vol. 12, no. 4, pp. 975-987, 2018.

[12] T. Acar and G. Ulusoy, "Approximation by modified SzászDurrmeyer operators," Periodica Mathematica Hungarica, vol. 72, no. 1, pp. 64-75, 2016.

[13] T. Acar, S. A. Mohiuddine, and M. Mursaleen, "Approximation by $(p, q)$-Baskakov-Durrmeyer-Stancu operators," Complex Analysis and Operator Theory, vol. 12, no. 6, pp. 14531468, 2018.

[14] K. J. Ansari, M. Mursaleen, and S. Rahman, “Approximation by Jakimovski-Leviatan operators of Durrmeyer type involving multiple Appell polynomials," Revista de la Real Academia de Ciencias Exactas, Físicas y Naturales. Serie A. Matemáticas, vol. 113, no. 2, pp. 1007-1024, 2019.

[15] A. Kajla and T. Acar, "A new modification of Durrmeyer type mixed hybrid operators," Carpathian Journal of Mathematics, vol. 34, no. 1, pp. 47-56, 2018.

[16] V. N. Mishra, K. Khatri, L. N. Mishra, and Deepmala, "Inverse result in simultaneous approximation by BaskakovDurrmeyer-Stancu operators," Journal of Inequalities and Applications, vol. 2013, no. 1, 2013.

[17] M. Mursaleen, S. Rahman, and K. Ansari, "Approximation by Jakimovski-Leviatan-Stancu-Durrmeyer type operators," Filomat, vol. 33, no. 6, pp. 1517-1530, 2019.

[18] M. Mursaleen and T. Khan, "On approximation by Stancu type Jakimovski-Leviatan-Durrmeyer operators," Azerbaijan Journal of Mathematics, vol. 7, no. 1, pp. 16-26, 2017. 
[19] M. Mursaleen and M. Nasiruzzaman, "Approximation of modified Jakimovski-Leviatan-Beta type operators," Constructive Mathematical Analysis, vol. 1, no. 2, pp. 88-98, 2018.

[20] S. A. Mohiuddine, T. Acar, and M. A. Alghamdi, "Genuine modified Bernstein-Durrmeyer operators," Journal of Inequalities and Applications, vol. 2018, no. 1, 2018.

[21] M. Qasim, M. Mursaleen, A. Khan, and Z. Abbas, "Convergence of generalized Lupaş-Durrmeyer operators," Mathematics, vol. 8, no. 5, 2020.

[22] B. Simsek, "Formulas derived from moment generating functions and Bernstein polynomials," Applicable Analysis and Discrete Mathematics, vol. 13, no. 3, pp. 839-848, 2019.

[23] Y. Simsek, "Q-beta polynomials and their applications," Applied Mathematics \& Information Sciences, vol. 7, no. 6, pp. 2539-2547, 2013.

[24] A. D. Gadzhiev, "A problem on the convergence of a sequence of positive linear operators on unbounded sets, and the theorems that are analogous to P. P. Korovkin's theorem," Doklady Akademii Nauk SSSR, vol. 218, pp. 1001-1004, 1974.

[25] P. P. Korovkin, Linear Operators and Approximation Theory, Hindustan Publishing Corporation, Delhi, 1960.

[26] A. Holhos, "Quantitative estimates for positive linear operators in weighted spaces," General Mathematics, vol. 16, no. 4, pp. 99-110, 2008.

[27] R. A. DeVore and G. G. Lorentz, "Constructive Approximation," in Grundlehren der Mathematischen Wissenschaften, Fundamental principales of Mathematical Sciences, SpringerVerlag, Berlin, 1993. 